\title{
Realistic and Efficient Radio Propagation Model for V2X Communications
}

\author{
Rashid Hafeez Khokhar ${ }^{1}$, Tanveer Zia $^{1}$, Kayhan Zrar Ghafoor ${ }^{2}$, Jaime Lloret ${ }^{3}$ and Muhammad \\ Shiraz ${ }^{4}$ \\ ${ }^{1}$ Charles Sturt University, Wagga Wagga, NSW 2678, Australia \\ ${ }^{2}$ Faculty of of Engineering, Koya University, Koya, Kurdistan Region-Iraq \\ ${ }^{3}$ Universidad Politecnica de Valencia, Camino de Vera s/n, 46022, Valencia, Spain \\ ${ }^{4}$ Faculty of Computer Science and Information Technology, University Malaya, Malaysia, \\ *Corresponding author: Rashid Hafeez Khokhar [e-mail: rkhokhar@csu.edu.au]
}

Received May 1, 2013; revised July 3, 2013; accepted July 20, 2013; published August 30, 2013

\begin{abstract}
Multiple wireless devices are being widely deployed in Intelligent Transportation System (ITS) services on the road to establish end-to-end connection between vehicle-to-vehicle (V2V) and vehicle-to-infrastructure (V2I) networks. Vehicular ad hoc networks (VANETs) play an important role in supporting V2V and V2I communications (also called V2X communications) in a variety of urban environments with distinct topological characteristics. In fact, obstacles such as big buildings, moving vehicles, trees, advertisement boards, traffic lights, etc. may block the radio signals in V2X communications. Their impact has been neglected in VANET research. In this paper, we present a realistic and efficient radio propagation model to handle different sizes of static and moving obstacles for V2X communications. In the proposed model, buildings and large moving vehicles are modeled as static and moving obstacles, and taken into account their impact on the packet reception rate, Line-of-sight (LOS) obstruction, and received signal power. We use unsymmetrical city map which has many dead-end roads and open faces. Each dead-end road and open faces are joined to the nearest edge making a polygon to model realistic obstacles. The simulation results of proposed model demonstrates better performance compared to some existing models, that shows proposed model can reflect more realistic simulation environments.
\end{abstract}

Keywords: Obstacles modelling, V2X communications, information dissemination in VANET, computer networks, radio propagation model 


\section{Introduction}

$\mathbf{V}_{\mathrm{e}}$ ehicular Ad hoc NETworks (VANETs) are emerging as a new promising field of wireless technology, which aims to deploy V2V and V2I communications (alco called V2X) for different applications such as roadway safety, dynamic routing planning, mobile sensing, incar entertainment, and even Internet access [1]. VANETs provide true ubiquitous communication networks with great features such as self configuration, infrastructureless, and rapidly deployable networks. Because of such promising applications and features, the automotive industry and the international standard organizations are paying special attention to VANETs research for obstacles handling [2,3], security [4], routing protocols [5, 6, 7, 8, 9], and mobility models [10]. But, in this paper we only focus on obstacles handling that is particularly challenging because the radio signals of high speed vehicles are obstructed by different static and moving obstacles. The antenna used in these vehicles have limited communication range and the signal may be weakened by these obstacles.

Most widely used stochastic radio propagation models (e.g., Free Space [11], TwoRayGround Reflection [11], Rayleigh Fading model [12], Ricean Fading model [13], Shadowing model [14], Log-distance Path Loss [11], mix ones [15, 16, 17]) rely on the overall statistical properties of the environment. Free Space model is not consider obstacles in city environments and the received signal power is based on three factors: the sender and receiver distance, antenna gains, and the transmitted power. Two-Ray Ground model demonstrates better performance than the Free Space model. Received signal strength can be predicted from long distance. However, two-ray is not concerned with the height and width of the nodes. It only assumes the received energy, which is the sum of direct LOS path and reflected path from the ground. In fact, there are different sizes of vehicles (i.e., cars, trucks, buses, and vans) on the roads. In Rayleigh Fading model, the indirect path between the transmitter and receiver nodes are taken into account in the Rayleigh model. Rayleigh Fading model is only suitable for wireless channels that have no LOS component. However multipath components may vary in amplitude and phase. The random multipath components are added to the LOS and can be seen as a Direct Current (DC) component in the random multipath in Rayleigh distribution. Similarly, the Ricean model only focuses on a single exact path and multiple reflected signals. Rayleigh and Ricean Fading are considering fast fading and caused by scattering while slow fading follows log-normal shadowing and occurs due to reflections of hill, building and obstacle. Similarly, in Shadowing model, the radio signals are set to some particular values, which make it not suitable for real urban environments. In general, these models are unable to provide satisfactory accuracy for distinctive urban vehicular environments as they do not consider any obstacle.

Recent VANET research [2,3] have confirmed that large moving vehicles also significantly affect vehicle-2-X communication in urban environments. The LOS between two communicating vehicles (cars) runs a high risk of interruption due to the presence of large vehicles such as buses and trucks. To develop a realistic radio propagation model for highly dynamic VANET is computationally challenging, and moving obstacles makes them more complicated. Cheng et al. [18] performed a narrow-band measurements of moving $\mathrm{V} 2 \mathrm{~V}$ radio propagation channel using $5.9 \mathrm{GHz}$ frequency band. In this experiment, the authors used slope piecewise linear channel model and observed that large moving vehicles are the major cause of reduction in received signal power. The geometry-based stochastic models $[19,20,21]$ are derived from a predefined stochastic distribution of effective scatterers by applying the fundamental laws of wave propagation. Such models can be easily adapted to different scenarios by changing the shape of the scattering region. However, 
models proposed in [19] are not able to learn the effect of vehicular traffic density on channel statistics. In addition, the wideband in these models has no capability to study pertap channel statistics. Similarly, only single-bounced rays were considered in Karedal's model [20] due to the fairly low vehicular traffic density of the measurements. However, the drifts of scatterers into different delay bins can be handled easily but with higher complexity. In another attempt, extensive measurements is reported in [22] which analysed the two ways high and low traffic densities of urban, suburban, and highways scenarios. The experimental results showed significantly different $\mathrm{V} 2 \mathrm{~V}$ channel properties using $5-\mathrm{GHz}$ band in low and high traffic conditions. Sen and Matolak [22] proposed several statistical channel models based on these measurements for different parameters such as delay spread, amplitude statistics, and correlations for multiple V2V environments. However, the main problem with these models is the limited $\mathrm{V} 2 \mathrm{~V}$ environment and traffic density.

We address the aforementioned problems by proposing a realistic and efficient radio propagation model to handle static and moving obstacles for V2X communications with the following desiderata:

I. How to develop a realistic and efficeint radio propagation model for meaningful VANETs simulation in urban environment which includes the presence of big buildings and large moving vehicles.

II. How the different shapes of static and moving obstacles in urban environments should be modelled to affect radio propagation signals with diffraction, attenuation, refraction, and reflection.

III. The intersections play an important role in VANETs simulations, how the sharp edges of obstacles at intersections should be identified for high data packet delivery.

With the above mentioned desiderata, we propose a realistic and efficient radio propagation model which takes into account the sharp edges of obstacles at intersections that are normally ignored in recently developed models. Two types of obstacles such as static (buildings) and moving (large vehicles) in urban environments are considered to be more realistic examples of obstacle modelling. Instead of fixing the radio signals to some particular radio range for all obstacles sizes and shapes, which is an overly optimistic attempt to reflect system performance in the real world, more accurate obstacles model is developed by considering all static and moving obstacles that can have an effect of radio signal propagation with diffraction, attenuation, refraction, and reflection. Federal Communication Commission (FCC) establishes rules to specify the frequencies of wireless LANs and the output power on each of those radio frequencies.

The rest of this paper is organized as follows. Section 2 describes previous work on Radio Propagation Models (RPMs) to handle static and moving obstacles for V2X communications. Section 3 introduces the system operation for our model. The proposed radio propagation model to handle obstacles by showing its design, providing an example in urban VANETs scenario, and optimizations, are formally presented in Section 4. In Section 5, after describing evaluation methodology, we present the performance analysis of the proposed RPM with some existing models using realistic city maps with static and moving obstacles. The paper is concluded in Section 5.

\section{Related Work}

Recently, several models [23, 24, 25] have been proposed to handle large obstacles such as buildings, floors, walls, etc. in urban areas, and trees, plants, etc. in rural areas. Buildings in 
urban environment are the main reason to obstruct the radio signal in V2X communication. Mahajan et al. [26] designed a model similar to the Two-Ray Ground model by including the influence of obstacles and the distance attenuation for the $802.11 \mathrm{~b}$ environment. In this model, real urban data is used to determine the impact of various obstruction factors on radio signals. The empirical data consists of two parameters such as distance from access point and signal strength. Signal strength variation was measured by placing an $802.11 \mathrm{~b}$ Linksys wireless access point at the corner of the building blocks. The authors also used Wavemon tool [27] to measure the signal strength from various locations near the building. However, this model work as a Two-Ray Ground model by simply considering an obstacles' distance and signal strength. Also, the performance evaluation of this model is measured using few number of nodes which is not enough to claim a reasonable simulation setup. A good and more realistic simulation for an urban environment should double this number. Radio Propagation Model with Obstacles (RPMO) proposed in [23] assumes the square shapes are city buildings and the distance from one building to another is the same. These buildings obstruct the radio signals between two vehicles on parallel streets. However, Martinez et al. [28] observed that the PRMO behaves like Two-Ray model if there are no obstacles. Also the most important parameter, distance attenuation, is not taken into account.

In another attempt, three RPMs such as Distance Attenuation Model (DAM), Building Model (BM), and Building and Distance Attenuation Model (BDAM) [28] proposed to handle static obstacles in urban environments. First the DAM considered two different possibilities including (1) the detailed analytical model to determine the signal strength and noise level of receiver node by bit and packet error rates, and (2) directly relate the bit or packet error rate with the distance under specific channel conditions to simplify the calculation and significantly reduce the simulation time. In BM, the communications between vehicles are only possible when they have clear line-of-sight with low penetration ratio. Finally, DAM and BM are combined to develop BDAM for realistic RPM with obstacles. These models claim better accuracy compared to Two-Ray Ground model in terms of warning messages and packet delivery ratio. In these models the sizes of the buildings are fixed from $10 \mathrm{~m}-40 \mathrm{~m}$ for different scenarios. However, the buildings have different sizes (width and height) in real-life urban environments.

Martinez et al. [29] proposed another scheme, Real Attenuation and Visibility (RAV), by combining attenuation and visibility schemes. In the attenuation scheme of RAV, a probability density function is used to determine the probability of packet successfully received at any given distance. Then, visibility scheme determines the existence of obstacles between two vehicles. In this scheme, BDAM is enhanced by adapting the real city map that contains streets and intersections. Attenuation scheme shows better simulation results using warning notification time and average blind vehicles. However, the performance of visibility scheme is lower than other schemes.

Otto et al. [2] investigated the impact of realistic RPM on VANET-based systems. IEEE 802.11b signal propagation measurements are collected in V2V communication for urban, suburban, and open road scenarios. Even the experiments are conducted on static obstacles (buildings) but the experimental results are the same on open roads during peak working hours and late night. The mean path loss exponent of 3.31 and Shadowing deviation of 4.84 $\mathrm{dB}$ were measured during peak working hours, while in late night the mean path loss and Shadowing deviations were measured as 3.1 and $3.23 \mathrm{~dB}$ respectively. Moving obstacles are the main cause of radio signal obstruction in two different experimental scenarios. Similarly, a wideband multiple-input-multiple-output model [20] proposed, which is based on extensive measurements performed in suburban and highway scenario using $5.2 \mathrm{GHz}$ frequency band. 
They proposed a generalization of geometry-based stochastic channel model techniques to distinguish between discrete and diffuse scattering contributions.

Boban et al. [3] proposed a model to satisfy essential requirements for V2V communication, such as realistic mobility patterns, accurate positioning, controllable complexity, and realistic radio propagation characteristics. The moving vehicles are considered as physical three dimensional obstacles that affect to the $\mathrm{V} 2 \mathrm{~V}$ communication. Extensive measurements were conducted to analyse the overall impact on the LOS obstruction, packet reception rate, and received signal power. The performance of the proposed model is evaluated by using the two real-world datasets on highway through stereoscopic aerial photography. The model clearly shows that moving vehicles obstruct the radio signals that generate major attenuation and packet loss. However, as the model has only been tested on the highway scenario, it needs extensive measurements to determine the impact of obstructing moving vehicles for vehicular communications in different urban and suburban scenarios with low, medium, and high node densities for both $2.4 \mathrm{GHz}$ and 5.9 $\mathrm{GHz}$ frequency bands.

\section{System Operation}

In this section, we describe the system operation to handle static and moving obstacles in VANETs. In order to know which neighbors are appropriate to contact with and process the realistic RPM, we have developed the following protocol.

A vehicle sends a broadcast message, only those vehicles and infrastructure devices in its radio range will be able to reply with the reply message. In this paper, we have used the beacon messages to broadcast messages to all neighbor vehicles. There are different broadcasting methods are used, such as periodically broadcast, ALOHA or pure ALOHA [30, 31]. These methods used tagged user approach to analyze a finite-user finite-buffer slotted Aloha system considering Additive White Gaussian Noise (AWGN) and frequency selective fading effects respectively. After broadcasting message, vehicle performs our proposed realistic RPM algorithm. The algorithm decides which are the most appropriate vehicle and infrastructure devices to connect with. These will be tagged as neighbors. Finally, it sends a V2X communication message to all selected neighbors. These steps are shown in Fig. 1.

For each reply received from a vehicle or infrastructure device at the same and parallel streets, the proposed system processes the realistic RPM algorithm. It operates as follows.

First it enters the propagation process, in this process vehicles broadcast a massage to all

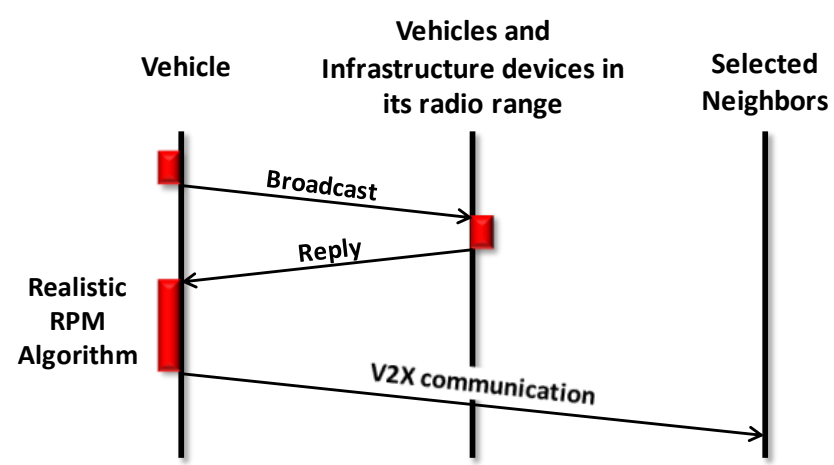

Fig. 1. Message flow of the system protocol.

neighbors within a communication range including vehicles on the same road segments and 
parallel streets. Signal to Interference plus Noise Ratio (SINR) is calculated and test either it is greater than or equal to certain threshold value. The Free Space propagation model is used to predict received signal strength, Shadowing model is used to express the obstructions in propagation environment by several physical effects, and the Fading model is used to jointly represent large-scale and small-scale effects. We use all these values to calculate SINR on the receiver side. In the second step obstacles are identified and modelled as different polygon shapes. Initially, buildings with different polygon shapes are considered as static obstacles and model obstacles using real map of city is to calculate the line segments and the centre of each obstacle. Similarly, it is assumed that the non-transmitting large vehicles (i.e., buses) are moving obstacles that may also obstruct radio signals. We have identified the static and moving obstacles by checking the sizes of obstacles, as the length and width of buses (moving obstacles) are same (i.e., $12 \mathrm{~m}$ and $3 \mathrm{~m}$ respectively) for all scenarios. If two vehicles intersect fixed size (i.e., $12 \mathrm{~m}$ and $3 \mathrm{~m}$ ) of length and width then such obstacles are moving obstacles (buses). On the other hand, the buildings (static obstacles) have different length and width and those buildings are identified as static obstacles. It is really possible that a building has same size of bus. Now, determine the SINR at the receiver side then check the condition if obstacle intersects the connection between vehicle two vehicles. The third step is to calculate a Maximum Interference Distance (MID) between all neighbor nodes and transmitting node. Considering the obstacles to compute network connectivity makes MID connection more specific and additional attenuation imposed by all obstacles that obstruct the connection. Fig. 2 illustrates the flowchart of proposed RPM to handle static and moving obstacles.

\section{Radio Propagation Model to handle Obstacles}

In this section, we propose the radio propagation model to handle static and moving obstacles in VANETs. The model consists of three steps including Propagation, Modeling Obstacles, and Maximum Interference Distance. In this section we describe these steps in more detail. The notations used in the proposed RPM algorithm are defined in Table 1. 


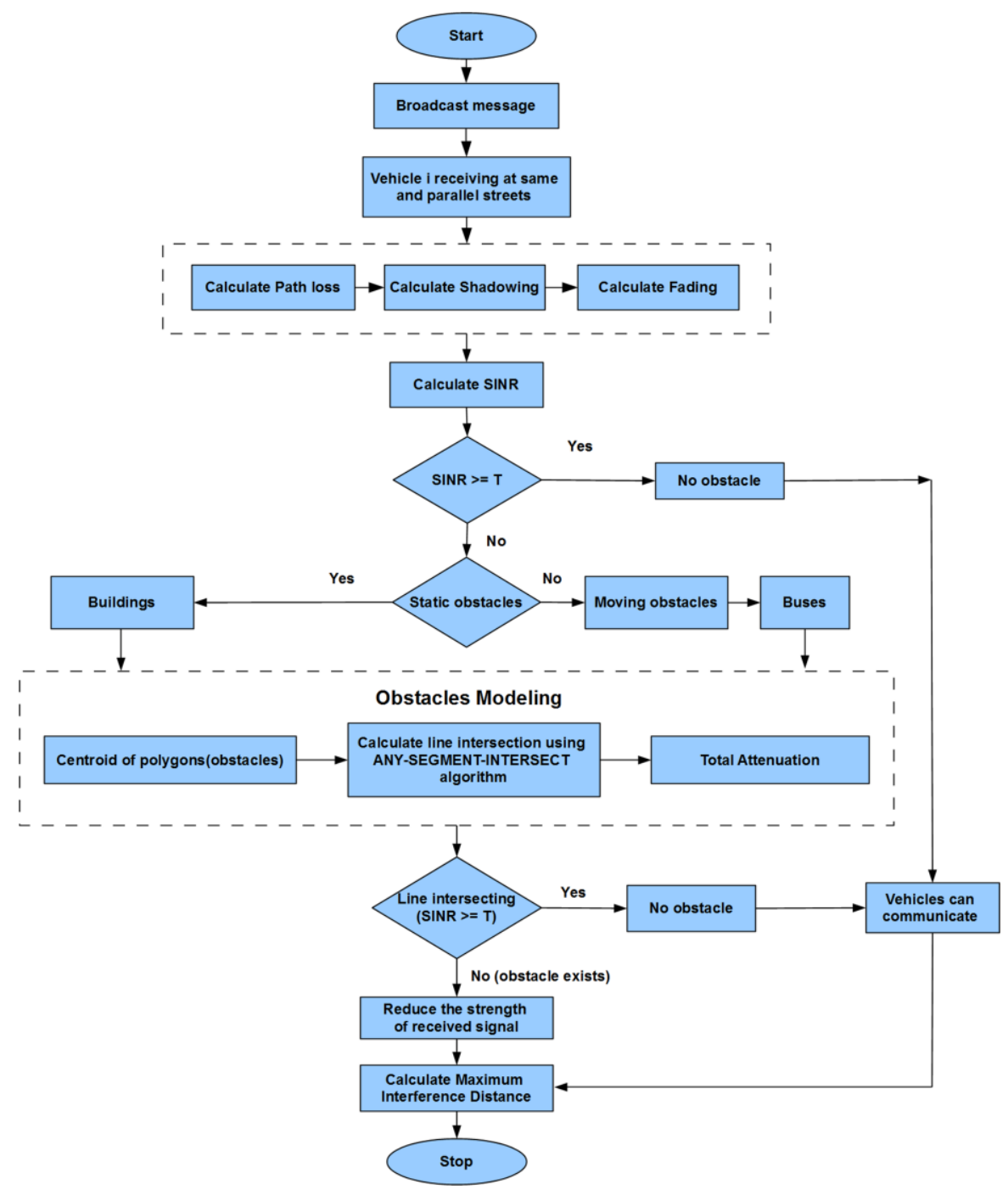

Fig. 2. Flowchart of proposed realistic radio propagation model algorithm

Table 1. Notations used in radio propagation model to handle obstacles

\begin{tabular}{|c|l|c|l|}
\hline Parameter & \multicolumn{1}{|c|}{ Description } & Parameter & \multicolumn{1}{|c|}{ Description } \\
\hline \hline$T$ & $\begin{array}{l}\text { Attenuation threshold required for successful } \\
\text { reception }\end{array}$ & $G_{t}$ & Transmitter antenna gains \\
\hline$N_{i}$ & The number of neighbors of vehicle $i$ & $G_{r}$ & Receiver antenna gains \\
\hline$N V_{i}$ & The set of vehicle $V_{i}$ neighbors & $\lambda$ & Wave length \\
\hline$L_{i}$ & The location of vehicle $i$ & $d_{i j}$ & Distance between node $i$ to node $j$ \\
\hline$r$ & The radio range for every vehicle & $O_{k}$ & Obstacle \\
\hline$P_{i}$ & Transmitted signal power & $S_{k}^{i j}$ & Set of line segments \\
\hline$N_{o}$ & Noise level & & \\
\hline
\end{tabular}




\subsection{Propagation}

Assume that vehicle $i$ broadcasts a message to the set of neighbors $N V_{i}$ in radio range including vehicles within the same road segment and on parallel streets. The set of neighbors for vehicle $i$ can be described as:

$$
N V_{i}=\{j \mid(\operatorname{dist}(i, j)<r) \quad i, j=1,2, \ldots ., n\}
$$

Where $\operatorname{dist}(i, j)$ is the distance between vehicles $i$ and $j$. Equation 1 describes, if vehicle $j$ is in the radio range of $i$, then $j$ is a neighbor of $i$.

It is also assumed that vehicle $i$ can communicate with neighbor vehicle $j$ from their respective locations, if SINR is greater than or equal to certain threshold $T$ (which is required for successful reception):

$$
\text { if }(\operatorname{SINR}(i, j) \geq T)
$$

The value of threshold $T$ is set according to the modulation used in the system. The SINR for transmission from vehicle $i$ to $j$ is computed as:

$$
\operatorname{SINR}(i, j)=\frac{P_{i} a(i, j)}{N_{0}+\sum_{k \neq i} P_{k} a(k, j)}
$$

where $a(i, j)$ is the path gain from transmitter $i$ to received $j$ and $P_{i}$ is the power transmitted by vehicle $i$. All neighbor nodes other than $i$ cause interference if they are simultaneously transmitting, contributing to the noise level $N_{0}$.

The Free Space propagation model is used to predict received signal strength from vehicle $i$ to $j$. The following well-known Friis equation [11] describes how the signal transmitted from $i$ to $j$ decays over distance $d_{i j}$ in Free Space:

$$
a_{P L}(i, j)=\frac{P_{i} G_{t} G_{r} \lambda^{2}}{(4 \lambda)^{2} d^{2}{ }_{i j} L}
$$

Where $G_{t}$ and $G_{r}$ describe the transmitter and receiver antenna gains respectively, $L$ captures circuit losses, and $\lambda$ is the wave length. The following Shadowing model proposed in [11] expresses the obstructions in propagation environment by several physical effects, e.g. reflection, diffraction, and scattering. The stochastic model is used to describe the variations of signal strength, e.g. by a zero-mean Gaussian distributed random variable with standard deviations (log-normal Shadowing).

$$
a_{S H}(i, j)=20 \log \left(4 \pi d_{i j} / L\right)+10 n \log \left(d_{i j} / d_{0}\right)+X_{\sigma}
$$

Where $d_{0}$ is the close-in reference distance which is determined from measurements close to the transmitter, $n$ is the path loss exponent which indicates the rate at which the path loss increases with distance, and $X_{\sigma}$ is a zero-mean Gaussian distribution random variable (in $\mathrm{dB}$ ) with standard deviation $\sigma$ (also in $\mathrm{dB}$ ). 
Similarly, fading model $a_{F D}(i, j)$ is used which was first proposed in [32] and later improved in conjunction with the obstacle model in [33]. The values of the above mentioned propagation models are used to calculate the SINR on the receiver side:

$$
a(i, j)=a_{P L}(i, j) a_{S H}(i, j) a_{F D}(i, j)
$$

\subsection{Obstacles Modelling}

The obstacles $O_{k} \in O$, where $k$ is the identity number of obstacles modelled as different polygon shapes. In most of the existing RPMs [28, 33, 34], only rectangular shapes are considered as obstacles. However, there are different types of shapes (as obstacles) in real world urban maps, as shown in Fig. 3.

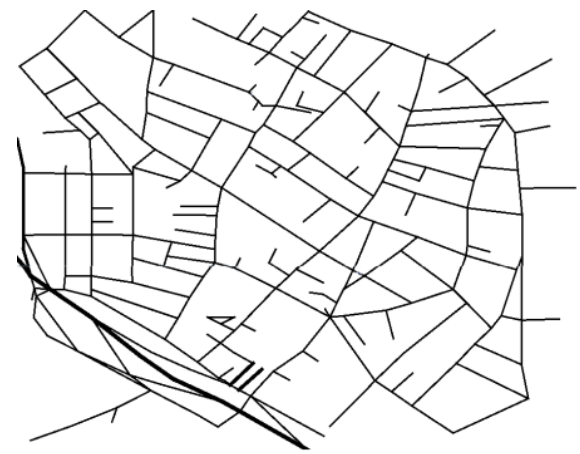

(a) Suffolk city map from Tiger Line DB used in simulation scenario with obstacles

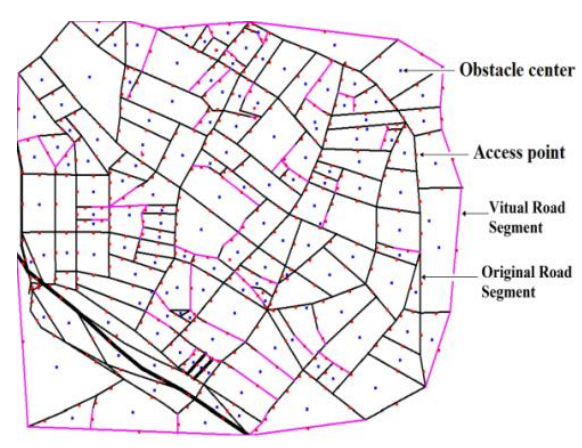

(b) Suffolk city map with virtual road segments and access points

Fig. 3. Original and modified Suffolk city maps

This research focuses on the following two types of obstacles including static and moving obstacles. Any static or moving obstacle $O_{k}$ can affect the transmission between two vehicles, if any border of an obstacle intersects with the line segment of the transmitting vehicle $i$ to its neighbor vehicle (receiver) $j$. First, buildings with different polygon shapes are considered as static obstacles. The first step to model obstacles is to calculate the line segments and the centre of each obstacle $O_{k}$. A Suffolk city map is used which is extracted from the TIGER/Line database of the US Census Bureau [35], as shown in Fig. 3(a)-3(b). However, the original map is not suitable for designing an obstacle model as the map consists of many dead-end roads, unsymmetrical shapes, and shapes with open faces. In the proposed RPM, all dead-end roads and open faces are joined with the nearest edge or road segment, as depicted in Fig. 3(b). Such road segments are presupposed as virtual road segments. Note that, when the simulation starts, the nodes will not move on the virtual road segments. It is only designed to calculate the center of each obstacle and radio propagation. In addition, the access points are fixed in the middle of each street, as shown in Fig. 3(b). The line segments of each polygon shape is determined using city map road segments. Now, the centroid $O_{k}\left(X_{c}, Y_{c}\right)$ of each obstacle is determined using the following equations: 


$$
\begin{gathered}
A=\frac{1}{2} \sum_{i=o}^{N-1}\left(x_{i} y_{i+1}-x_{i+1}+y_{i}\right) \\
X_{c}=\frac{1}{N A} \sum_{i=0}^{N-1}\left(x_{i}+x_{i+1}\right)\left(x_{i} y_{i+1}-x_{i+1} y_{i}\right) \\
Y_{c}=\frac{1}{N A} \sum_{i=0}^{N-1}\left(y_{i}+y_{i+1}\right)\left(x_{i} y_{i+1}-x_{i+1} y_{i}\right)
\end{gathered}
$$

where $A$ is the area and $N$ is the number of vertices of obstacle $O_{k}$.

Similarly, it is assumed that the non-transmitting large vehicles (i.e., buses) are moving obstacles that may also obstruct radio signals. An urban scenario with two cars and bus is illustrated in Fig. 4. If the moving obstacles are on the streets, then only the corner points of the obstacles are needed to calculate a unit interval of 0.1 seconds. However, it would be more complicated to handle moving obstacles at intersections. In this case, the moving obstacles can rotate at any angle from $O^{0}$ to $360^{\circ}$.

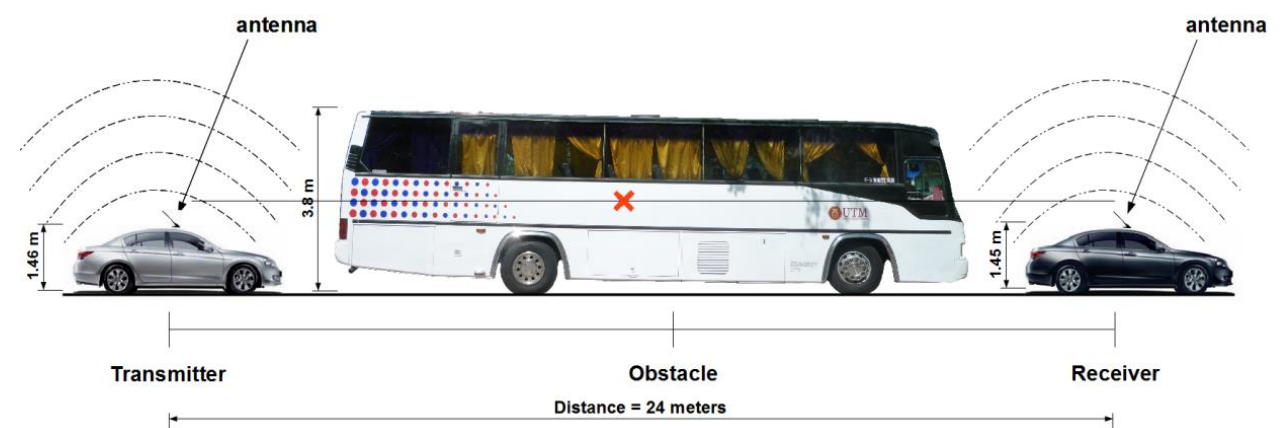

Fig. 4. Bus as moving obstacle between two cars in urban scenario

Fig. 5(a) and Fig. 5(b) show if the obstacle is turning at an intersection with angle $\beta_{k}$, then for every corner point $(x, y)$ at first position (non-rotated) of obstacle, corresponding to corner points $\left(x^{\prime}, y^{\prime}\right)$ of second position (after rotation) is calculated as:

$$
\begin{aligned}
& x^{\prime}=x_{c}\left(o_{k}\right)+\left(x-x_{c}\left(o_{k}\right)\right) \cos \beta_{k}-\left(y-y_{c}\left(o_{k}\right)\right) \sin \beta_{k} \\
& y^{\prime}=y_{c}\left(o_{k}\right)+\left(x-x_{c}\left(o_{k}\right)\right) \sin \beta_{k}-\left(y-y_{c}\left(o_{k}\right)\right) \cos \beta_{k}
\end{aligned}
$$

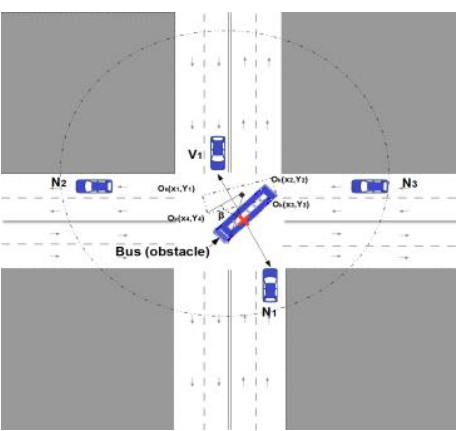

(a) Moving Obstacles at Intersection 00

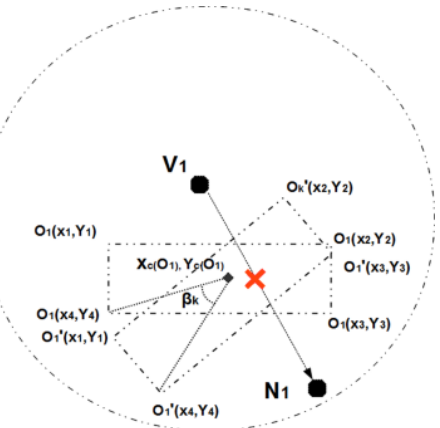

(b) Moving obstacles rotated with angle $\beta_{k}$

Fig. 5. Moving obstacles scenario 
For example, Fig. 6 shows static and moving obstacles with 5 and 4 line segments respectively. ANY-SEGMENTS-INTERSECT algorithm proposed in [11] is used to test whether any line of polygon shape intersects the moving vector from vehicle $V_{i}$ to set of neighbor $N_{j}$. In this case, a set of line segments is needed to compute for all possible moving vectors $S_{k}^{i j}$ as:

$$
S_{k}^{i j}=\left\{\bar{V}_{i} N_{j}, \forall \overline{O_{k}\left(x_{e}, \overline{y_{e}}\right) O_{k}\left(\bar{x}_{e+1}, y_{e+1}\right)}: 1 \leq e \leq n\right\}
$$

where $n$ is the number of obstacle points. A static obstacles scenario is described in Fig. $\mathbf{6}$,

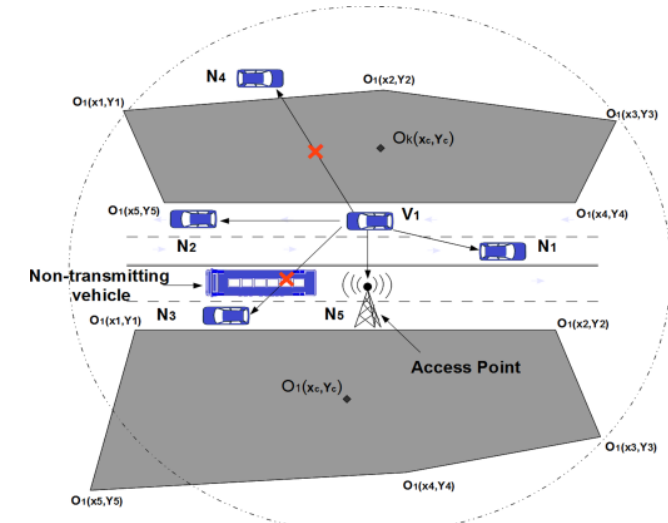

Fig. 6. Static (buildings) and moving (bus) obstacles in urban vehicular scenario

where vehicle $V_{1}$ initially broadcasts a message to all neighbors $\left(N_{1}, N_{2}, N_{3}, N_{4}, N_{5}\right)$ within the same and parallel streets. Assume that $N_{5}$ is the access point to forward real-life traffic information to infrastructure networks. Vehicles $N_{1}, N_{2}$, and $N_{3}$ are on the same street, the vehicles $N_{1}$ and $N_{2}$ can communicate easily, but $N_{3}$ is obstructed by a moving obstacle. In addition, $N_{4}$ is on a parallel street and can not communicate because the obstacle line segment intersects the moving vector of vehicle $V_{1}$ to $N_{4}$. It means $S_{k}^{i j}$ is true and the attenuation $a_{k}$ of the obstacle $O_{k}$ further reduces the strength of the received signal. The total attenuation caused by all obstacles within communication range of node $i$ can be calculated as:

$$
a_{O B}(i, j)=\prod_{O_{k} \in O} a_{k}^{b_{k}^{i j}}
$$

where the $b_{k}^{i j}$ indicates whether the obstacles $O_{k}$ intersects and it can be calculated as:

$$
b_{k}^{i j}=\left\{\begin{array}{l}
1 \quad \text { if ANY-SEGMENTS - INTERSECT } \quad\left(S_{k}^{i j}\right) \text { is True } \\
0 \text { otherwise }
\end{array}\right.
$$

Now, determine the SINR at the receiver side by putting the value of $a_{O B}(i, j)$ in equation 3 
then check the condition in equation 2 if obstacle intersect the connection between vehicle $V_{i}$ and set of neighbor $N_{j}$.

\subsection{Maximum Interference Distance (MID)}

It has already been discussed in literature that the signal sent by one node affects all nodes within radio range. Faraway nodes may receive low signals and can easily be negligible in sensitive VANET simulations. The solution to handling such problem is to calculate a Maximum Interference Distance (MID) between all neighbor nodes and transmitting node. The MID is calculated by Friss equation [11]:

$$
d_{I}=\sqrt[\alpha]{\frac{P_{\max }}{P_{\min }}\left(\frac{\lambda}{4 \pi}\right)^{2}}
$$

where $P_{\min }$ is the minimum power at receiver node required for interference and $P_{\max }$ maximum power is being transmitted.

In VANET simulation, the presence of obstacles in the propagation environment may shield nodes from interfering with each other. The additional attenuation imposed by the obstacle affects the MID in this case. The obstacles shielding effect may cause nodes to be disconnected, increasing the chance of the hidden node problems, identified in [34]. The shielding effects must be considered when computing the MID to reduce the chance of hidden terminal problem. Considering the obstacles to compute network connectivity makes MID connection more specific. Next equation calculates MID where $P_{\max }$ is proportional to the additional attenuation imposed by all obstacles that obstruct the connection.

$$
d_{I}^{i j}=\sqrt[\alpha]{a_{O B}(i, j) \frac{P_{\max }}{P_{\min }}\left(\frac{\lambda}{4 \pi}\right)^{2}}
$$

\section{Performance Evaluation}

The performance of proposed RPM is evaluated using two main types of urban scenarios including static and moving obstacles. First in static obstacle, the proposed realistic RPM is compared with the RPMO [23], BDAM [29], and No obstacles scenario. In the following, the simulation setup, evaluation metrics, and the experimental results using with obstacles scenario are presented.

\subsection{Simulation Setup}

A map of Suffolk city with a simulation dimension of $940 \mathrm{~m} \times 750 \mathrm{~m}$ were used in all experiments and the area contains 322 road segments with a total length of 701528.75 meters. As it has already discussed in a previous section, the original map is modified to generate accurate attenuation at the center of each obstacle. The sizes of the static obstacles are between 20-400 meters wide. Table 2 describes the simulation parameters used in all experiments. The integrated, configurable, and scalable Swans++ simulator [37] has been used to evaluate the performance of the proposed RPM to handle obstacles for V2X communications. This simulator is widely used for our purposes because of its realistic mobility model in US cities [38]. Both macro-benchmark and micro-benchmark results run 
full on Swans++ simulations highlighting the throughput and memory advantages of Swans++. The number of vehicles and obstacles (static and moving) are varied in urban environment to evaluate the performance of proposed RPM for different scenarios. The total simulation time for single flow was 300 seconds, however, the first 60 seconds of simulation are discarded to get more accurate results. The selection of routing protocols are equally important to test our obstacles model, we used two different routing protocols such as position-based (Greedy Perimeter Stateless Routing (GPSR) [39]) and topology-based (Adhoc On-demand Distance Vector (AODV) [40]) routing protocols A STreet RAndom Waypoint (STRAW) mobility model [10] is used for node mobility. The STRAW offers realistic vehicular mobility, contains an efficient car following and lane changing model, and a real-time traffic controller over actual Suffolk city map imported from TIGER/LINE database [36]. The 10 random source and destination nodes of pairs using Constant Bit Rate (CBR) and User Datagram Protocol (UDP)-based packet are used in each experiment. We used the IEEE 802.11 with DCF standard at MAC layer for the wireless configuration. Similarly, the Free Space, Shadowing and Fading propagation models used with $250 \mathrm{~m}$ communication range at physical layer to characterize physical propagation. In addition, the values of exponent for path loss formula and standard deviation for lognormal shadow fading set to 2.8 and 6.0 respectively.

Table 2. Parameter values used in simulation for proposed radio propagation model

\begin{tabular}{|l|l|l|l|}
\hline \multicolumn{1}{|c|}{ Parameter } & \multicolumn{1}{c|}{ Value } & \multicolumn{1}{c|}{ Parameter } & \multicolumn{1}{c|}{ Value } \\
\hline Simulation dimension & $940 \mathrm{~m} \times 750 \mathrm{~m}$ & Transmission range & $250 \mathrm{~m}$ \\
\hline Simulation area & $701528.75 \mathrm{~m}^{2}$ & Simulation time & $300 \mathrm{~s}$ \\
\hline Number of static obstacles & 146 & Vehicle speed & $20-80 \mathrm{~m} / \mathrm{h}$ \\
\hline Number of moving obstacles & $25,50,75$ buses & Routing protocols & GPSR, AODV \\
\hline Number of vehicles & $100,150,200$ & Mobility model & STRAW \\
\hline Access points & 322 & Radio frequency & $2.4 \mathrm{GHz}$ \\
\hline Building width & $20-400 \mathrm{~m}$ & MAC protocol & IEEE $802.11 \mathrm{~b}$ DCF \\
\hline Packet sending frequency & 1 per second & & \\
\hline
\end{tabular}

\subsection{Metrics}

The performance of the proposed radio propagation model to handle static and moving obstacles was evaluated by varying the number of nodes and moving obstacles. The standard metrics used to assess the performance are the following:

- Packet Delivery Ratio: The Packet Delivery Ratio (PDR) calculates the number of data packets sent by the source node and how many data packets (in \%) the destination node successfully received.

- Delivery Latency: The delivery latency calculates the total time a message was posted by the source to destination node. The average delay characterizes the latency generated by the routing protocols.

\subsection{Simulation Results in Urban Environment (Static Obstacles Scenario)}

The experimental results present average values of evaluation metrics over a number of executions with randomly generated mobility scenarios and random placement of vehicles on the map. The following five experiments were run using the aforementioned simulation setup.

In the first experiment warning notification time vs. number of vehicles receiving warning messages is calculated by repeating the experiment for 10 runs. The numbers of static and moving obstacles are 146 and 50 respectively. Fig. 7(a)-7(c) illustrate that the vehicles 
received warning messages by varying the number of nodes 100, 150, 200 nodes for different RPMs. In all scenarios, the proposed RPM shows better performance when less number of vehicles received the warning messages is between $63 \%$ and $76 \%$. With 100 nodes (as shown in Fig. 7(a)), the warning messages do not reach all vehicles, 50\% of the vehicles receive the warning messages within 0.20 seconds. However, RPMO and BDAM is about $25 \%$, and No obstacles is about $50 \%$ more vehicles received the messages within same time period as compared to the proposed RPM. Similarly, the RPMO and BDAM received more messages compared to the proposed model in the case of 150 and 200 nodes, as depicted in Fig. 7(b)-7(c). The reason for the low performance in cases of more vehicles is that the RPMO does not consider attenuation distance. Also, in the No obstacles scenario the maximum number, $96 \%$ of the vehicles received the warning messages within 1 second, which is $20 \%$ higher than the proposed RPM. The main reason for these results is the implementation of more restrictive obstacles model, as fewer vehicles receive messages since messages are obstructed by buildings and non-transmitting vehicles.

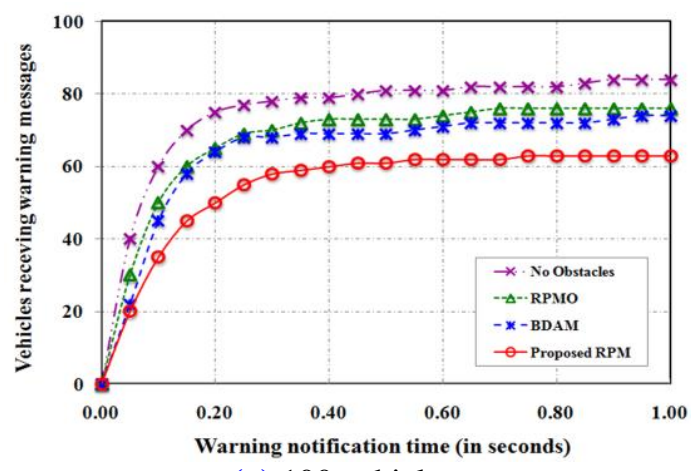

(a) 100 vehicles

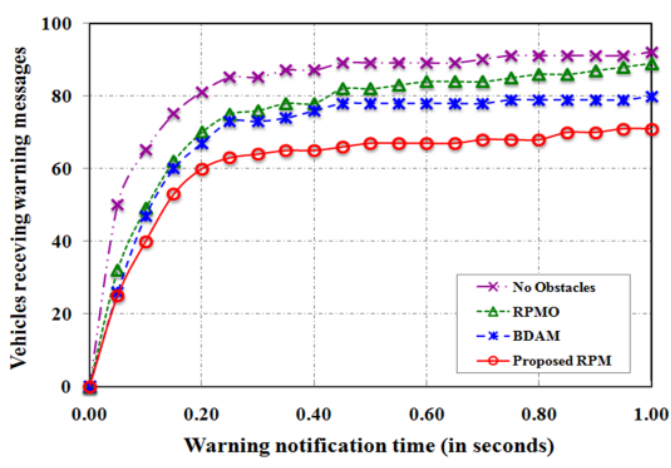

(b) 150 vehicles

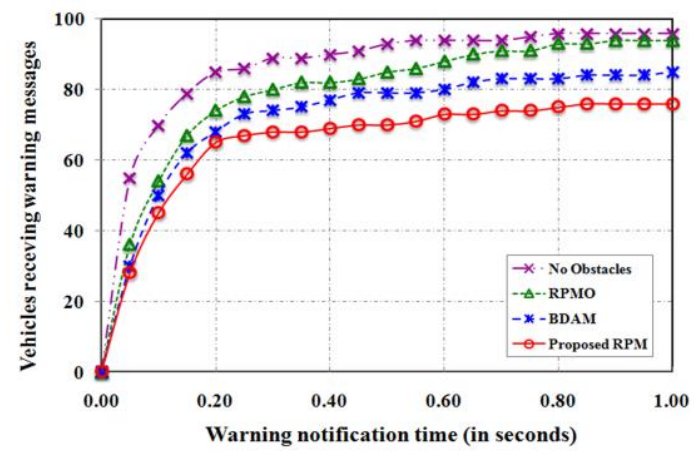

(c) 200 vehicles

Fig. 7. CDF of vehicles receiving the warning message for proposed RPM, RPMO, BDAM, and no obstacles

In the second experiment the performance of the proposed RPM is evaluated using the evaluation metric packet delivery ratio. This experiment was again repeated for 10 runs by manually inserting 100, 150, and 200 random nodes. As shown in Fig. 8(a)-8(c), the PDR's of all RPMs are consistently higher when the vehicle density is increased. In the first scenario, Fig. 8(a), the proposed model receives about $40 \%$ less packets as compared to No obstacles scenario, and compared to RPMO and BDAM models, the difference is about $15 \%$ and $20 \%$ respectively. In the second scenario, Fig. 8(b) the PDR's of proposed RPM is about $50 \%$ which is clearly less than other models. Similarly in Fig. 8(c), in last scenarios, more 
than $90 \%$ of the data packets were received by the destination node in cases of No obstacles, which is distinctly higher than the proposed model. However, when the packet rates were between 2.5 and 5.0 per second the PDR of the proposed model is unexpectedly reduced from $58 \%$ to $40 \%$.

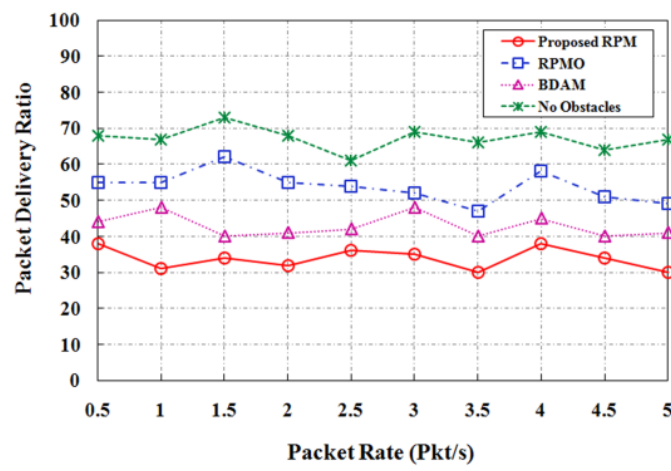

(a) 100 vehicles

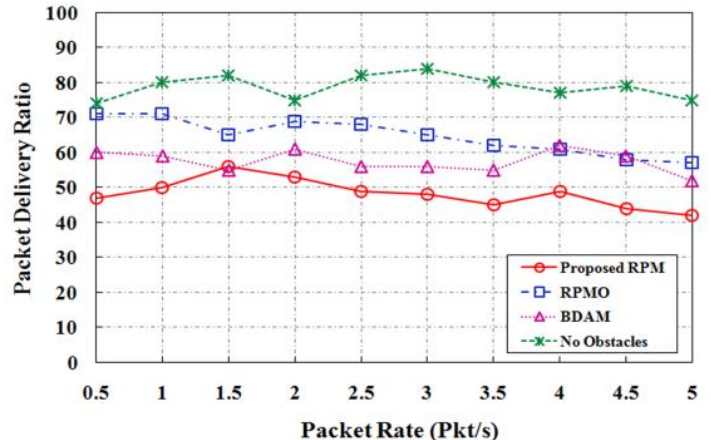

(b) 150 vehicles

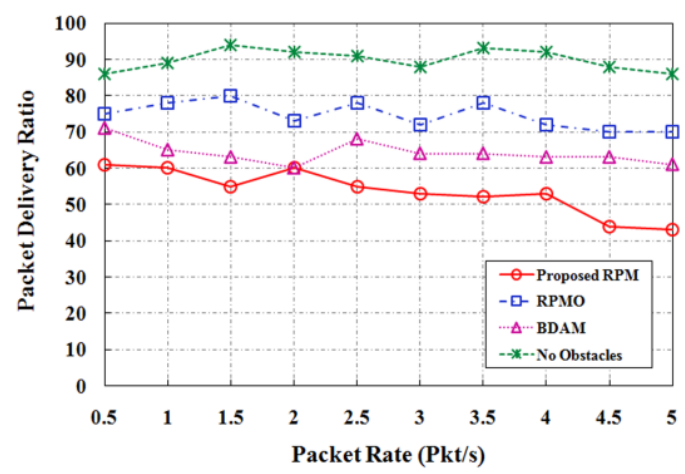

(c) 200 vehicles

Fig. 8. Experimental results of PDR using position-based routing protocol (GPSR) for proposed RPM, RPMO, BDAM, and no obstacles

The reason for this result is the source and destination node may not be reachable because the GPSR routing protocol was used and the nodes may have reached local maximum and are trying to recover using perimeter mode. In all cases that PDR increases when packet rates increase, which shows the protocols can transfer more data packets in the network. The PDR's of all protocols are less accurate in these experiments as more accurate RPM is used to handle obstacles, limiting the radio signals in the wireless networks.

In third experiment, the PDR is measured using topology-based routing protocol (AODV). In this experiment, same numbers of nodes were used to repeat 10 runs. Fig. 9(a)-9(c), the PDR's of all RPMs are consistently higher but lower than the second experience where we used position-based routing protocol. As discussed in literaure, the topology-based routing protocols do not work well in VANET due to their dynamism. Routes break as soon as they are established by route reply packers. On the other hand the position-based routing protocols like GPSR gets rid of route query and route reply altogether. 


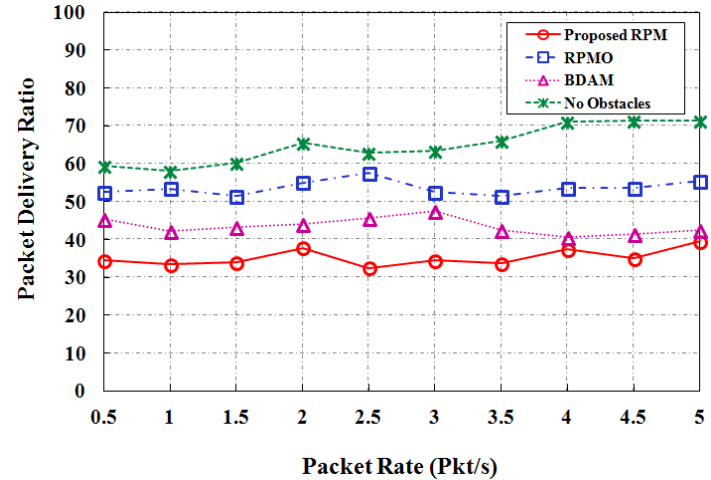

(a) 100 vehicles

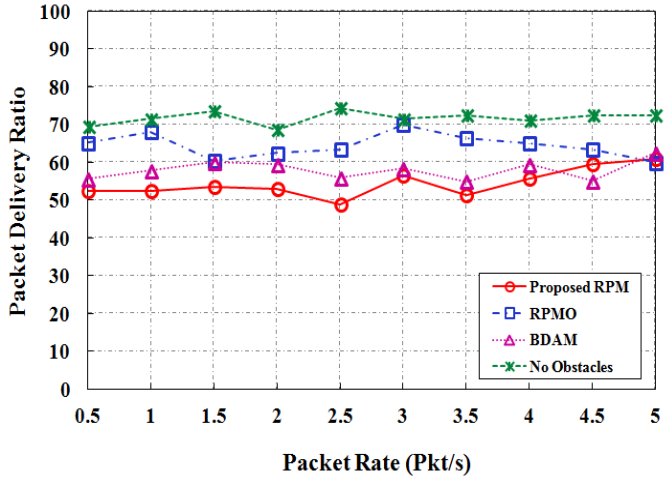

(b) 150 vehicles

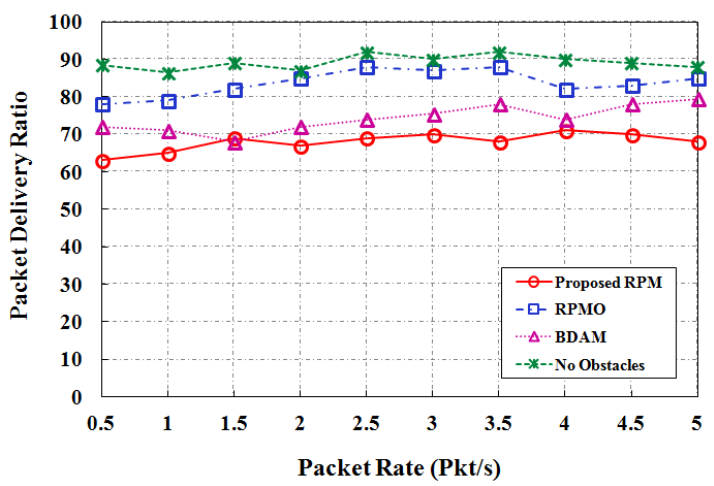

(b) 150 vehicles

Fig. 9. Experimental results of PDR using topology-based routing protocol (GPSR) for proposed RPM, RPMO, BDAM, and no obstacles

In the foruth and fifth experiments, the performance is evaluated using delivery latency for position-based and topology-based routing protocols using only 150 nodes, as shown in Fig. 10(a) and Fig. 10(b). The results for network density for 100 and 200 nodes were almost the same and only 150 nodes were used in these experiments. Fig. 10(a) shows that the latency using position-based routing protocol (GPSR) for all models decreases when node densities are increased. The main reason is that the routes remain active for longer periods of time as the number of nodes increases. The source node repairs the route and fewer packets need to be buffered. It can also be observed that the data packets take more time in the proposed RPM as compared to other models. The main reason for the longer delay is that the vehicles have to travel for a longer distance to find the next hop due to more restrictive proposed RPM. Similarly, Fig. 10(a) shows that the delivery latency using topology-based routing protocol (AODV) for all models are higher than position-based routing protocol (GPSR). As discussed earlier the link breaks frequently in topology-based routing protocol and route maintenance takes longer time, especially in case of with obstacles scenario. 


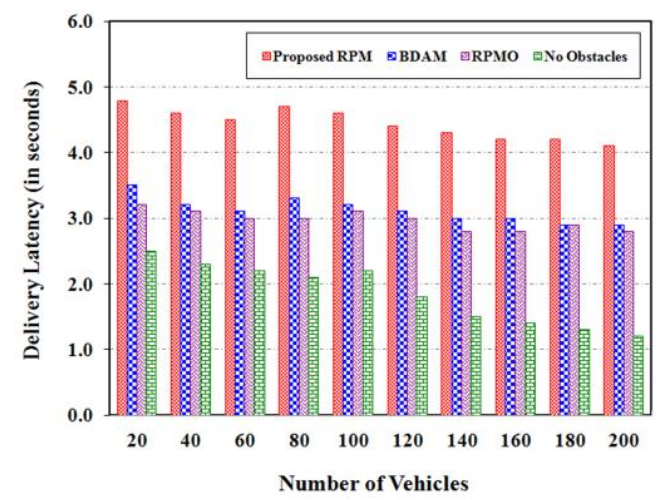

(a) Delivery latency vs different node density using position-based routing protocol (GPSR)

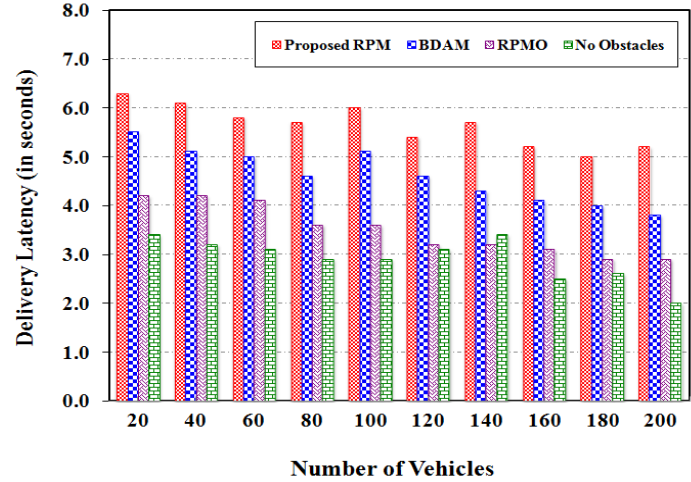

(b) Delivery latency vs different node density using topology-based routing protocol (AODV)

Fig. 10. Delivery latency of proposed RPM, RPMO, BDAM, and no obstacles (static obstacles scenario) using position-based and topology-based routing protocols

\subsection{Simulation Results in Urban Environment (Moving Obstacles Scenario)}

Finally, the RPM is analyzed using the delivery latency which is the most straightforward method for evaluating the performance of application in case of LOS (no obstruction) and non-LOS (25, 50, and 75 obstructing buses) using 100 and 200 nodes. In both scenarios the Cumulative Distribution Function (CDF) curves do not reach 1 because not all messages were received at their destination. It is clear that neither LOS nor non-LOS parameter settings will yield realistic results. Fig. 11 (a) shows the LOS parameter has higher latency using 100 nodes with a median delivery latency of 84.5 seconds as there were no obstacles to block LOS. The non-LOS case exhibits a larger variation for 25, 50, and 75 obstacles and these three distributions are overall significantly different, thus clearly showing the impact of the obstructing bus. Similarly, median delivery latency is lower for no obstacles, which is around 71.5 in case of 200 nodes and non-LOS cases are also significantly lower.

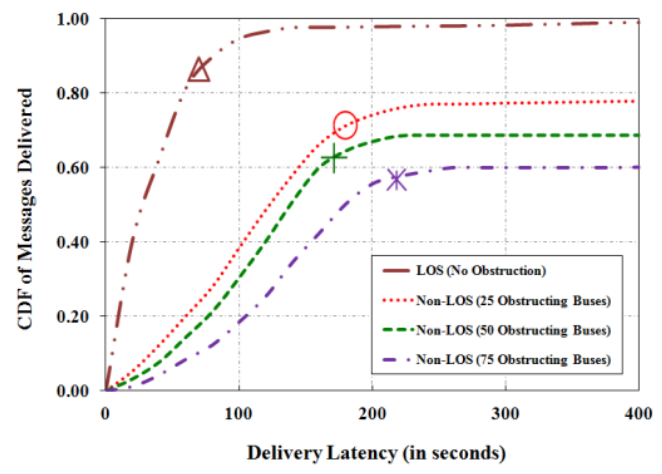

(a) CDF of delivery latency using 100 nodes

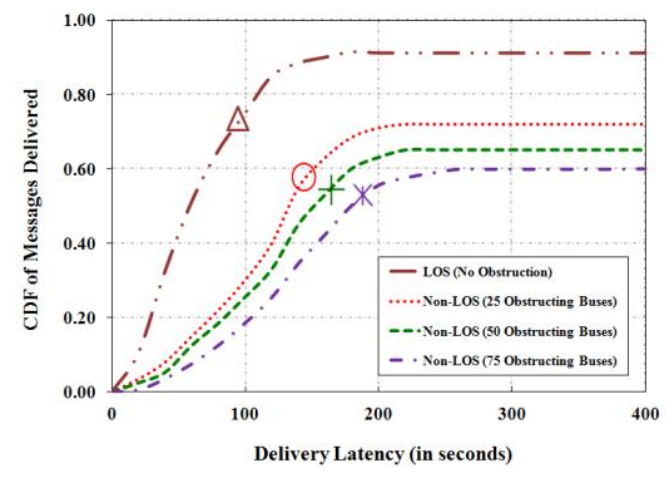

(b) CDF of delivery latency using 200 nodes

Fig. 11. CDF of delivery latency in case of LOS (no obstruction) and non-LOS (obstructing bus) for urban environment 


\section{Conclusion}

The most widely used RPMs are not designed to handle different sizes of obstacles in urban environment. The radio signals are set to a particular value based on the width of buildings and assumes that all buildings are equal in sizes. However, in reality, obstacles are numerous sizes such as small or big buildings, non-transmitting vehicles (trucks, buses, van, etc.), advertisement boards, trees, etc. In this paper, we propose a realistic RPM to handle static and moving obstacles in real-life V2X communications. In the proposed RPM, a more accurate mechanism is used to generate radio signals with diffraction, attenuation, refraction, and reflection. The simulation results conclude that the performance of proposed RPM is better as compared to other RPMs in terms of warning messages received by each vehicle, PDR, delivery latency, and path length. Obviously, the more restrictive and accurate obstacle model blocks the signals in many cases.

In future works we will introduce the realistic model in other routing protocols for VANETS such as GeoDTN, GeOpps, VADD, CBF, To-Go, GPCR, etc., and we will compare the performance of our system when there is large or small environments [41].

\section{References}

[1] J. Lloret, A. Canovas, A. Catalá, M. Garcia, "Group-based Protocol and Mobility Model for VANETs to Offer Internet Access," Journal of Network and Computer Applications, Available online March 2012. DOI: 10.1016/j.jnca.2012.02.009

[2] J.S. Otto, F.E. Bustamante, and R.A. Berry, "Down the block and around the corner the impact of radio propagation on inter-vehicle wireless communication," in Proc. of 29th IEEE International Conference on Distributed Computing Systems, (ICDCS '09), Montreal, Quebec, Canada. June 22-26, 2009. DOI: 10.1109/ICDCS.2009.60

[3] M. Boban, T.T.V. Vinhoza, M. Ferreira, J. Barros, and O.K. Tonguz, "Impact of vehicles as obstacles in vehicular ad hoc networks," IEEE Journal on Selected Areas in Communications, 29(1):15 -28, 2011. http://dx.doi.org/10.1109/JSAC.2011.110103

[4] S.U. Rehman, W.-C. Song, and G.-L. Park, "Associativity-based on-demand multi-path routing in mobile ad hoc networks," KSII Transactions on Internet and Information Systems, Vol.3 No.5, pages 475-491, 2009. http://dx.doi.org/10.3837/tiis.2009.05.004

[5] M.-W. Ryu, S.K.S.-H. Cha, J.-G. Koh, and K.-H. Cho. "Position-based routing algorithm for improving reliability of inter-vehicle communication," KSII Transactions on Internet and Information Systems, Vo. 5, Pp. 1388 - 1403, 2011. http://dx.doi.org/10.3837/tiis.2011.08.002

[6] R. H. Khokhar, M. A. Ngadi, M. S. Latiff, and M. A. Amin, "Reactive traffic-aware routing strategy for urban vehicular environments," International Journal of Ad Hoc and Ubiquitous Computing, Vol. 10, No. 3 (2012). DOI: 10.1504/IJAHUC.2012.048625

[7] R. H. Khokhar, R. M. Noor, K. Ghafoor, C.-H. Ke, and M. A. Ngadi, "Fuzzy-assisted socialbased routing for urban vehicular environments," EURASIP Journal on Wireless Communications and Networking, 2011(1):178, 2011. http://dx.doi.org/10.1186/1687-14992011-178

[8] K.Z. Ghafoor, K. Bakar, S.A. Salleh, K. Lee, M. Mohamad, M. Kamat, and M. Arshad, "Fuzzy logic-assisted geographical routing over vehicular ad hoc networks," International Journal of Innovative Computing Information and Control, Vol. 8, Number 7(B), July 2012 pp. 5095-5120.

[9] K.Z. Ghafoor, K.A. Bakar, K. Lee, H. AL-Hashimi, "A Novel Delay- and Reliability- Aware Inter-Vehicle Routing Protocol" Network Protocols and Algorithms, Vol. 2, No 2. Pp.66-88. 2010 http://dx.doi.org/10.5296/npa.v2i2.427 
[10] D. R. Choffnes and Fabián E. Bustamante, "An integrated mobility and traffic model for vehicular wireless networks," in Proc. of the 2nd ACM international workshop on Vehicular ad hoc networks, Cologne, Germany, September 2, 2005. Pp. 69-78. http://dx.doi.org/10.1145/1080754.1080765

[11] T. S. Rappaport, "Wireless Communications Principles and Practice," Prentice Hall, 2nd edition edition, 2002.

[12] R. H. Clarke, "A statistical theory of mobile radio reception," Bell Systems Technical Journal, 47 (6):9571000, 1968.

[13] S.O. Rice, "Mathematical analysis of random noise," Bell Telephone Laboratories, 1944. PMid:18015998 PMCid:PMC1625059

[14] M. Gudmundson, "Correlation model for shadow fading in mobile radio systems," Electronics Letters, 27(23):2145 -2146. Nov. 1991. http://dx.doi.org/10.1049/el:19911328

[15] J. Lloret, J. Tomás, M. Garcia, A. Cánovas, “A Hybrid Stochastic Approach for Self-Location of Wireless Sensors in Indoor Environments,” Sensors, Vol. 9, Issue 5, Pp. 3695-3712. May 2009. http://dx.doi.org/10.3390/s90503695

[16] Y. Zou, J. Zhu, B. Zheng, Y. Yao, “An Adaptive Cooperation Diversity Scheme With Best-Relay Selection in Cognitive Radio Networks," IEEE Transactions on Signal Processing, Vol. 58, No. 10, October 2010 http://dx.doi.org/10.1109/TSP.2010.2053708

[17] Y. Zou, Y. Yao, B. Zheng, "Diversity-Multiplexing Tradeoff in Selective Cooperation for Cognitive Radio," IEEE Transactions on Communications, Vol. 60, No. 9, September 2012. 10.1109/TCOMM.2012.072612.110180

[18] L. Cheng, B.E. Henty, D.D. Stancil, F. Bai, and P. Mudalige, "Mobile vehicle-to-vehicle narrowband channel measurement and characterization of the $5.9 \mathrm{GHz}$ dedicated short range communication frequency band," IEEE Journal on Selected Areas in Communications, 25(8):1501 -1516, 2007. http://dx.doi.org/10.1109/JSAC.2007.071002

[19] A.G. Zajic, G.L. Stuber, T.G. Pratt, and S.T. Nguyen, "Wideband mimo mobile-to-mobile channels: Geometry-based statistical modeling with experimental verification," IEEE Transactions on Vehicular Technology, 58(2):517 -534, feb. 2009. http://dx.doi.org/10.1109/TVT.2008.928001

[20] J. Karedal, F. Tufvesson, N. Czink, A. Paier, C. Dumard, T. Zemen, C.F. Mecklenbrauker, and A.F. Molisch, "A geometry-based stochastic mimo model for vehicle-to-vehicle communications," IEEE Transactions on Wireless Communications, 8(7):3646-3657, 2009. http://dx.doi.org/10.1109/TWC.2009.080753

[21] K. Mammasis and P. Santi, "A two-dimensional geometry-based stochastic model," IEEE Transactions on Wireless Communications, 11(1):38 -43, January 2012. 21. 10.1109/TWC.2011.111211.110671

[22] I. Sen and D.W. Matolak, "Vehicle-vehicle channel models for the 5-ghz band," IEEE Transactions on Intelligent Transportation Systems, 9(2):235 -245, 2008. http://dx.doi.org/10.1109/TITS.2008.922881

[23] S. Marinoni and H.H. Kari, "Ad hoc routing protocol performance in a realistic environment," in Proc. of International Conference on Systems and International Conference on Mobile Communications and Learning Technologies (ICN/ICONS/MCL 2006), 23-29 April 2006. 10.1109/ICNICONSMCL.2006.39. PMCid:PMC2065967

[24] J. Lloret, J.J. Lopez, C. Turro, and S. Flores, “A fast design model for indoor radio coverage in the 2.4 ghz wireless lan," in Proc. of 1st International Symposium on Wireless Communication Systems (ISWCS'04), Port Louis (Mauricio Island), September 20-22, 2004. pp 408-412. DOI:10.1109/ISWCS.2004.1407279

[25] J. Lloret, M. Garcia, D. Bri, and S. Sendra, “A wireless sensor network deployment for rural and forest fire detection and verification," Sensors, 9(11):8722-8747, 2009. http://dx.doi.org/10.3390/s91108722. PMid:22291533 PMCid:PMC3260610

[26] A. Mahajan, N. Potnis, K. Gopalan, and A. Wang, "Modeling vanet deployment in urban settings," in Proc. of 10th ACM/IEEE International Symposium on Modeling, Analysis and 
1952 Khokhar et al.: Realistic and Efficient Radio Propagation Model to Handle Obstacles for Vehicle-to-X Communications

Simulation of Wireless and Mobile Systems, Chania, Crete Island, Greece, October 2007. doi=10.1.1.132.7195

[27] Wavemon Website. At: http://packages.debian.org/unstable/net/wavemon

[28] F.J. Martinez, Chai-Keong Toh, J.-C. Cano, C.T. Calafate, and P. Manzoni, "Realistic radio propagation models (rpms) for vanet simulations," in Proc. of in Wireless Communications and Networking Conference (WCNC 2009), Budapest, Hungary, 5-8 April 2009. http://dx.doi.org/10.1109/WCNC.2009.4917932

[29] F.J. Martinez, M. Fogue, M. Coll, J. Cano, C.T. Calafate, and P. Manzoni, "Assessing the impact of a realistic radio propagation model on vanet scenarios using real maps," in Proc. of 9th IEEE International Symposium on Network Computing and Applications (NCA 2010), Cambridge, Massachusetts, USA. July 15-17, 2010, pp. 132 -139. DOI: 10.1109/NCA.2010.24

[30] Sanqing Hu ; Yu-Dong Yao ; Sheikh, A.U., Haleem, M.A., "Tagged user approach for finite-user finite-buffer S-Aloha analysis in AWGN and frequency selective fading channels," in Proc. of 34th IEEE Sarnoff Symposium, pp1-5, 2011. doi:10.1109/SARNOF.2011.5876474

[31] Sanqing Hu, Yu-Dong Yao, Sheikh, A.U., "Slotted Aloha for cognitive radio users and its tagged user analysis," in Proc. of 21st IEEE WOCC, pp1-5, 2012 doi:10.1109/WOCC.2012.6198140

[32] H. S. Lichte, V. Stefan, K. Holger, A. Imad, L. Luis, and W. Joerg, "Design and evaluation of a routing-informed cooperative mac protocol for ad hoc networks," in Proc. of 27th Annual Joint Conference of the IEEE Computer Communication Societies (INFOCOM), Phoenix, USA, April 2008. DOI: 10.1109/INFOCOM.2008.249

[33] H.S. Lichte and J. Weide, "Modeling obstacles in inet/mobility framework: motivation, integration, and performance," in Proc. of 2nd International Conference on Simulation Tools and Techniques (Simutools '09), Rome, Italy, March 3-5, 2009. DOI: 10.4108/ICST.SIMUTOOLS2009.5680

[34] R. Nagel and S. Eichler, "Efficient and realistic mobility and channel modeling for vanet scenarios using omnet++ and inet-frameworkm," in Proc. of 1st Int. conference on Simulation tools and techniques for communications, networks and systems (Simutools '08), Marseille, France, March 03 - 07, 2008. doi=10.1.1.144.221

[35] Tiger. tiger/line and tiger-related products. U.S. census bureau. Available at: http://www.census.gov/geo/www/tiger/shp.html

[36] A. Köpke, M. Swigulski, K.Wessel, D.Willkomm, P. T. Klein Haneveld, T. E. V. Parker, O. W. Visser, H. S. Lichte, and S. Valentin, "Simulating wireless and mobile networks in omnet++ the mixim vision," in Proc. of 1st Int. conference on Simulation tools and techniques for communications, networks and systems (Simutools '08), Marseille, France, March 03 - 07, 2008. doi: 10.1016/S1389-1286(03)00356-6

[37] AquaLab. Swans++ - extensions to the scalable wireless ad-hoc network simulator, 2011. At: http://www.aqualab.cs.northwestern.edu/projects/143-swans-extensions-to-the-scalable-wirelessad-hoc-network-simulator

[38] Francisco J. Martinez, Chai Keong Toh, Juan-Carlos Cano, Carlos T. Calafate and Pietro Manzoni, "A survey and comparative study of simulators for vehicular ad hoc networks (VANETs)," Wireless Communications and Mobile Computing, Volume 11, Issue 7, pages 813828, July 2011. doi: $10.1002 / w c m .859$

[39] B. Karp and H. T. Kung, "Gpsr: greedy perimeter stateless routing for wireless networks," in Proc. of Sixth Annual International Conference on Mobile Computing and Networking (MobiCom 2000), Boston Massachusetts (USA). 6-11 August 2000. doi:10.1145/345910.345953

[40] Perkins, C.E.; Royer, E.M., "Ad-hoc on-demand distance vector routing," in Proc. of Second IEEE Workshop on Mobile Computing Systems and Applications (WMCSA '99), 25-26 Feb 1999. http://dx.doi.org/10.1109/MCSA.1999.749281

[41] M. Tarique, A. Hossain, R. Islam, C.A. Hossain, "Issues of Long-Hop and Short-Hop Routing in Mobile Ad Hoc Networks: A Comprehensive Study," Network Protocols and Algorithms, Vol. 2, No 2 (2010). Pp. 107-131 http://dx.doi.org/10.5296/npa.v2i2.430 


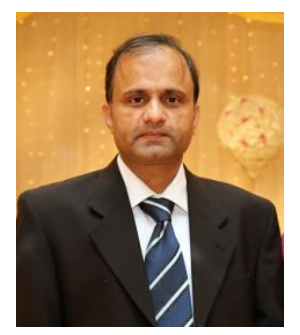

Rashid Khokhar is working as Teaching Fellow in the School of Computing \& Mathematics, Faculty of Business, Charles Sturt University (CSU). He received his $\mathrm{PhD}$ in Computer Science from University Technology Malaysia (Malaysia) in 2011. He also received his M.S in Computer Science (by research) from the same university in 2005. Earlier, he did his M.S (Information Technology) and M.S (Statistics) from Preston University (Pakistan) and University of the Punjab (Pakistan) in 2003 and 1999 respectively. He is the author of various articles and serving as member of editorial board and reviewers in international journals and conferences. He is a member of IEEE and ACM. His areas of interest are Vehicular \& Mobile Ad Hoc Network (Architectures, Protocols, Security, and Algorithms), Mobile Cloud Computing, Artificial Intelligence and Real-Time Communications, and High Performance Computing.

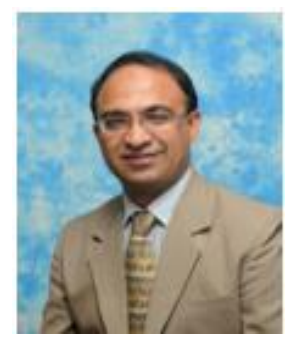

Tanveer Zia is a Senior Lecturer in Computing, Course Coordinator for the Doctor of Information Technology, and Associate Head of School, School of Computing \& Mathematics, Faculty of Business, Charles Sturt University (CSU). $\mathrm{He}$ has earned his $\mathrm{PhD}$ from the University of Sydney in 2008, Master of Interactive Multimedia (MIMM) from University of Technology Sydney in 2004, MBA from Preston University USA in 1997, and Bachelors of Science in Computer Sciences from Southwestern University, Philippines in 1992. Tanveer's broader research interests are in ICT security. Specifically he is interested in security of low powered mobile devices. He is also interested in biometric security, cyber security, cloud computing security, information assurance, protection against identity theft, trust management, forensic computing, and law and ethics in ICT. $\mathrm{He}$ is serving on Technical and Program Committees of several international conferences in his area of research. He actively publishes in international conferences, symposiums, workshops, and refereed journals.

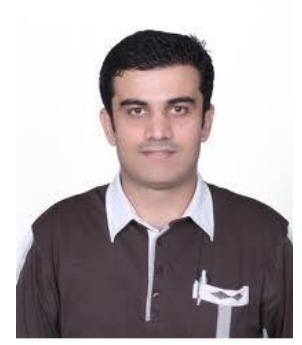

Kayhan Ghafoor received the BSc degree in Electrical Engineering from Salahaddin University, the MSc degree in Remote Weather Monitoring from Koya University and the PhD degree in Wireless Networks from University Technology Malaysia in 2003, 2006, and 2011,respectively. He is currently involved in organizing Committee of a workshop named Smart Sensor Protocols and Algorithms under The 9th International Conference on Mobile Ad-hoc and Sensor Networks (MSN-2014) which will be held in Hungary. He is serving as a member of Editorial Board of IJCSIS, IJAIT, IJACSA, JCIT and IJRRAN. He is also serving as an associate editor of IJNPA and IJANC. Kayhan is appeared as a TPC for many prestigious international conferences. He served as a reviewer for 20 reputed international journals. He awarded UTM Chancellor award in 2012, UTM International Doctoral Fellowship (IDF) and Kurdistan Regional Government (KRG) scholarship (Ahmad Ismail Foundation). My current research interests include routing over Vehicular Ad Hoc Networks and Tactical Wireless Networks, as well as Artificial Intelligence and network coding applications. He am a member of IEEE Vehicular Technology Society, IEEE Communications Society, and International Association of Engineers (IAENG). 

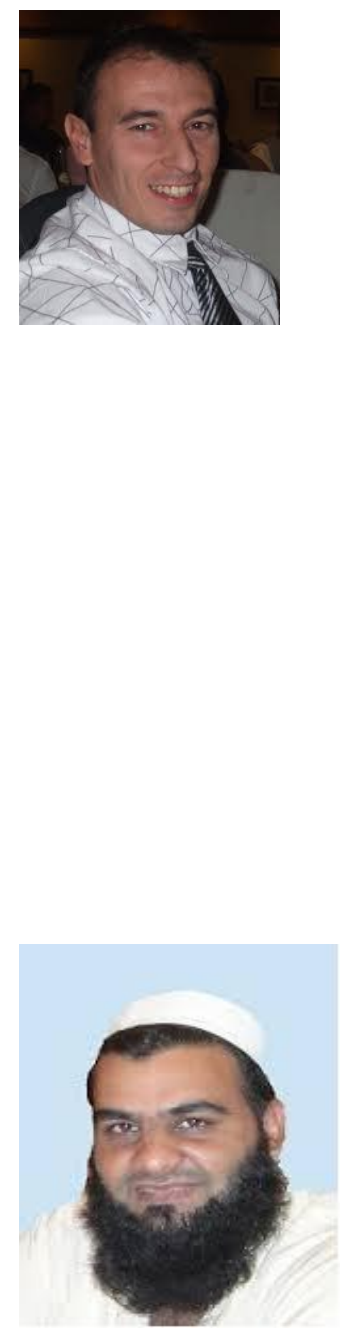

Prof. Jaime Lloret received his M.Sc. in Physics in 1997, his M.Sc. in electronic Engineering in 2003 and his Ph.D. in telecommunication engineering (Dr. Ing.) in 2006. He is a Cisco Certified Network Professional Instructor. He worked as a network designer and administrator in several enterprises. He is currently Associate Professor in the Polytechnic University of Valencia. He is the head of the research group "communications and remote sensing" of the Integrated Management Coastal Research Institute and he is the head of the "Active and collaborative techniques and use of technologic resources in the education (EITACURTE)" Innovation Group. He is the director of the University Expert Certificate, Redes y Comunicaciones de Ordenadores, the University Expert Certificate Tecnologías Web y Comercio Electrónico, and the University Master "Digital Post Production". He is currently Vice-chair of the Internet Technical Committee (IEEE Communications Society and Internet society). He has authored 12 books and has more than 220 research papers published in national and international conferences, international journals (more than 50 with ISI Thomson Impact Factor). He has been the co-editor of 15 conference proceedings and guest editor of several international books and journals. He is editor-in-chief of the international journal "Networks Protocols and Algorithms", IARIA Journals Board Chair (8 Journals) and he is associate editor of several international journals. He has been involved in more than 160 Program committees of international conferences and in many organization and steering committees. He led many national and international projects. He is currently the chair of the Working Group of the Standard IEEE 1907.1. He has been the general chair (or co-chair) of 18 International conferences. He is co-chairman AdHocNow 2014 and local chair IEEE Sensors 2014. He is IEEE Senior and IARIA Fellow.

Muhammad Shiraz is pursuing his $\mathrm{PhD}$ Candidature under Commonwealth Scholarship Program at Faculty of Computer and Information Technology University of Malaya, Malaysia. He is an active researcher in theMobile Cloud ComputingResearch Group at Faculty Computer Science and Information Technology University Malay Kuala Lumpur. He is also working as Assistant Professor at Department of Computer Science, Federal Urdu University of Arts, Sciences and Technology Islamabad, Pakistan. He completed under graduation from CECOS University Peshawar Pakistan with the distinction of Gold medal. He has Completed Masters in Computer Science from Allama Iqbal Open University Islamabad Pakistan in 2007. His areas of interest include distributed applications design for Ubiquitous Networks, Distributed Systems, Lightweight Applications, Smart Client Applications and Optimization Strategies, Mobile Cloud Computing. 